\title{
High-dose methotrexate for primary CNS lymphoma in the elderly
}

\author{
Siobhan Ng, ${ }^{1}$ Mark A. Rosenthal, David Ashley, and Lawrence Cher \\ Department of Medical Oncology and Clinical Haematology, Royal Melbourne Hospital, Parkville, Victoria, \\ Australia 3050 (S.N., M.A.R.); Department of Medical Oncology and Clinical Haematology, Royal Children's \\ Hospital, Parkville, Victoria, Australia 3050 (D.A); and Austin and Repatriation Medical Centre, Heidelberg, \\ Victoria, Australia 3181 (L.C.)
}

Primary central nervous system lymphoma (PCNSL) in the immunocompetent patient reaches a peak incidence in the sixth and seventh decades of life. This retrospective study reviewed the efficacy and tolerability of high-dose methotrexate (HDMTX) in an elderly patient population. Between May 1995 and September 1998, ten consecutive elderly patients with histologically proven PCNSL were treated with HDMTX. The median age was 72.5 years and eight patients $(80 \%)$ were older than 70 years. HDMTX was well tolerated with no episodes of grade 4 toxicity nor febrile neutropenia. Toxicity included grade 3 nausea (1), grade 2 mucositis (2), and grade 2 asymptomatic elevation of liver transaminases (2). Grade 1 toxicity occurred in three patients with nausea, diarrhea, and mild reversible elevation in serum creatinine in one patient each. Six patients had a complete response and three patients achieved a partial response, giving an overall response rate of $90 \% \quad(95 \%$ confidence interval, $56 \%-100 \%)$. The median overall survival for the cohort was 36 months (range 4-43 months). In summary, HDMTX is well tolerated in this elderly population with PCNSL and achieves response rates and median survival comparable with other chemotherapy or radiotherapy regimens. Neuro-Oncology 2, 40-44, 2000 (Posted to Neuro-Oncology [serial online], Doc. 99-24, December 13, 1999. URL <neuro-oncology.mc.duke.edu>)

Received 31 August 1999, accepted 18 October 1999

${ }^{1}$ Address correspondence and reprint requests to Siobhan Ng, M.D., Department of Medical Oncology and Clinical Haematology, Royal Melbourne Hospital, Parkville, Victoria, Australia 3050.

${ }^{2}$ Abbreviations used are as follows: ECOG, Eastern Cooperative Oncology Group; HDMTX, high-dose methotrexate; PCNSL, primary central nervous system lymphoma; PS, performance status.

\section{Introduction}

PCNSL $^{2}$ accounts for only $2 \%-5 \%$ of all primary brain tumors but appears to be increasing in incidence (Eby et al., 1988; Fine and Mayer, 1993; Lutz and Coleman, 1994; Rosenthal and Green, 1995). The rise in incidence is not simply attributable to human immunodeficiency virus infection but has also occurred in the immunocompetent population (Rosenthal and Green, 1995). PCNSL in the immunocompetent patient reaches a peak incidence in the sixth and seventh decades of life and is most commonly an intermediate grade B-cell lymphoma as defined by the Working Formulation (Rosenthal and Green, 1995).

The management of PCNSL remains ill-defined, with options including surgery, corticosteroids, irradiation, and chemotherapy. Extensive surgical resection has no role in the primary therapy of PCNSL. Murray et al. documented no difference in survival between those patients who underwent an extensive resection compared with those who received limited biopsy (Murray et al., 1986). Furthermore, the median survival in patients who underwent surgery alone was only 3 months (Murray et al., 1986). Corticosteroids may induce a partial or even complete remission, but this is rarely durable (Singh et al., 1982). Whole-brain irradiation has a clear beneficial effect in overall survival, achieving median survival times of 12-18 months (Berry and Simpson, 1981; Murray et al., 1986; Rosenthal and Green, 1995).

Most contentious is the role of chemotherapy in patients with PCNSL. There is debate about the use of chemotherapy, the agents used, the manner in which they are given, and the necessity for postchemotherapy irradiation (Rosenthal and Green, 1995). Established regimens include intrathecal methotrexate, chemotherapy delivered after blood-brain barrier disruption, single-agent highdose i.v. methotrexate, and standard multi-agent i.v. antilymphoma chemotherapy combinations (Blay et al., 1998; 
Brada et al., 1990; Cher et al., 1996; DeAngelis et al., 1992; Gabbai et al., 1989; Neuwelt et al., 1991; O’Neill et al., 1999; Rosenthal and Green, 1995; Rosenthal et al., 1993).

HDMTX was used by Cher et al. without postchemotherapy irradiation in an attempt to reduce neurotoxicity after therapy (Cher et al., 1996). They established that the regimen was well tolerated and achieved a $90 \%$ response rate with a median overall survival time of 15 months or more.

This report retrospectively examined the efficacy and tolerability of HDMTX in an elderly patient population. Patients older than 60 years have consistently been shown to have a poorer outcome on univariate and multivariate analyses (Blay et al., 1998; Nelson et al., 1992). It is of particular importance that we examine treatmentrelated outcomes in this age group because the peak incidence of PCNSL in the immunocompetent cohort includes this elderly population.

\section{Materials and Methods}

Between May 1995 and September 1998, ten consecutive elderly immunocompetent patients with histologically proven PCNSL were treated with HDMTX as first-line therapy based on the regimen of Cher et al. (1996). HDMTX was given by i.v. according to a three-phase protocol: $8 \mathrm{~g} / \mathrm{m}^{2}$ every 14 days for three cycles, $3.5 \mathrm{~g} / \mathrm{m}^{2}$ every 28 days for three cycles, and $3.5 \mathrm{~g} / \mathrm{m}^{2}$ for two cycles three months apart. The dose was reduced for renal impairment, hepatic dysfunction, or previous toxicity. Intravenous folinic acid rescue ( $30 \mathrm{mg}, 4$ times a day) was commenced $24 \mathrm{~h}$ after i.v. methotrexate and was continued until the daily plasma methotrexate level fell to $<0.1 \mathrm{mmol} / \mathrm{l}$. Adequate hydration was maintained throughout hospitalization, and the urine was alkalinized to a $\mathrm{pH}$ of $>7.0$ by using i.v. $\mathrm{NaHCO}_{3}$.

Full blood examination, electrolytes, urea, serum creatinine, and liver function tests were assessed before and during each cycle of treatment while the patient was hospitalized or as indicated. Physical examination and toxicity notation were performed at the commencement of each treatment cycle. Standard radiologic criteria for response and the World Health Organization criteria for toxicity were used. All patients who received HDMTX were assessed for response, toxicity, and survival.

\section{Results}

Patient characteristics are detailed in Table 1 . The patient population was elderly with a median age of 72.5 years. All patients were older than 60 years, and $8(80 \%)$ were older than 70 years. The ECOG performance status (Oken et al., 1982) of the group varied: ECOG 0-1 $(40 \%)$ and ECOG $2-3(60 \%)$. All patients were considered immunocompetent, although human immunodeficiency virus testing was not universally performed. One patient had been receiving low-dose methotrexate therapy for rheumatoid arthritis, and a second patient had the CREST syndrome.

\section{Toxicity}

HDMTX was well tolerated even in this elderly population. Toxicity data were recorded for the first phase of therapy $\left(8.5 \mathrm{~g} / \mathrm{m}^{2}\right.$ every 14 days $)$. This was the most intensive phase of treatment, and no new or additional toxicity was noted in any of the patients in subsequent treatment phases. There was no clinical or radiologic evidence of late toxicity related to HDMTX.

There were no episodes of grade 4 toxicity or febrile neutropenia. Toxicity included grade 3 nausea (one patient), grade 2 mucositis (two patients), and grade 2 asymptomatic elevation of liver transaminases (two patients). Grade 1 toxicity occurred in three patients: nausea, diarrhea, and mild reversible elevation in serum creatinine in one patient each. Two patients developed urinary tract infections related to urinary catheterization during treatment but were not neutropenic at the time. Both patients responded promptly to appropriate antibiotic therapy.

\section{Dose Intensity and Dose Delays}

Table 2 details the dose intensity and treatment delays for each patient during the first treatment phase. Factors that might affect dose intensity such as age, ECOG performance status, and glomerular filtration rates are also listed. Dose reduction occurred in three patients because of a reduction in glomerular filtration rate. None of

Table 1. Patient characteristics

\begin{tabular}{lc} 
Characteristic & Number $(\%)$ \\
\hline Sex & \\
Male & $5(50)$ \\
Female & $5(50)$ \\
Age & \\
$>70$ years & $8(80)$ \\
$\leq 70$ years & $2(20)$ \\
Performance status & \\
ECOG 0 & $1(10)$ \\
ECOG 1 & $3(30)$ \\
ECOG 2 & $3(30)$ \\
ECOG 3 & $3(30)$ \\
ECOG 4 & $0(0)$ \\
Site of lesion & \\
Supratentorial & $10(100)$ \\
Solitary & $5(50)$ \\
Multiple & $5(50)$ \\
CSF & \\
Positive cytology & $2(20)$ \\
Negative cytology & $4(40)$ \\
Not tested & $4(40)$ \\
Pathology and cell type & \\
B cell & \\
Cell lineage not stated & \\
Large cell & $(80)$ \\
\hline Absions: & \\
\hline
\end{tabular}

Abbreviations: ECOG, Eastern Cooperative Oncology Group; CSF, cerebrospinal fluid. 
Table 2. Dose intensity, dose delays, and response to HDMTX

\begin{tabular}{|c|c|c|c|c|c|c|c|c|c|c|}
\hline Patient & $\begin{array}{l}\text { Age } \\
\text { (yrs.) }\end{array}$ & $\begin{array}{l}\text { Pretreatment } \\
\text { ECOG PS }\end{array}$ & $\begin{array}{c}\text { Posttreatment } \\
\text { ECOG PS }\end{array}$ & $\begin{array}{l}\text { Dose } \\
(\%)\end{array}$ & $\begin{array}{c}\text { GFR } \\
(\% \\
\text { normal) }\end{array}$ & $\begin{array}{l}\text { Days to complete } \\
\text { first phase } \\
\text { (ideal = 29) }\end{array}$ & $\begin{array}{l}\text { Reason for } \\
\text { dose reduction } \\
\text { or dose delay }\end{array}$ & $\begin{array}{c}\text { Best response } \\
\text { to HDMTX }\end{array}$ & $\begin{array}{l}\text { Time to } \\
\text { progression } \\
\text { (months) }\end{array}$ & $\begin{array}{l}\text { Survival }{ }^{a} \\
\text { (months) }\end{array}$ \\
\hline 1 & 71 & 3 & 2 & 100 & 82 & 30 & Logistical & $\mathrm{CR}$ & 18 & $35+$ \\
\hline 2 & 73 & 1 & 1 & 100 & 64 & 51 & Mucositis & CR & 15 & 20 \\
\hline 3 & 75 & 2 & 1 & 75 & 50 & 27 & Reduced GFR & $C R$ & $40+$ & $40+$ \\
\hline 4 & 73 & 2 & 1 & 60 & 53 & 35 & Logistical & PR & 5 & 36 \\
\hline 5 & 74 & 3 & 2 & 100 & 83 & 38 & Mucositis & $\mathrm{CR}$ & 24 & 43 \\
\hline 6 & 66 & 1 & 1 & 100 & 60 & 34 & Logistical & $\mathrm{CR}$ & $5+$ & $5+$ \\
\hline 7 & 72 & 2 & 2 & 100 & 56 & 28 & Logistical & PR & 2 & 8 \\
\hline 8 & 75 & 3 & 2 & 100 & 64 & 70 & Patient refusal & $\mathrm{CR}$ & 19 & 20 \\
\hline 9 & 68 & 0 & 0 & 50 & 50 & 32 & Reduced GFR & PR & $7+$ & $7+$ \\
\hline 10 & 72 & 1 & 1 & 100 & 70 & $\begin{array}{c}\text { Ceased after } \\
\text { phase } 1\end{array}$ & $\begin{array}{c}\text { No clinical } \\
\text { improvement }\end{array}$ & SD & NA & 4 \\
\hline
\end{tabular}

Abbreviations: HDMTX, high-dose methotrexate; ECOG, Eastern Cooperative Oncology Group; PS, performance status; GFR, glomerular filtration rate; CR, complete response; PR, partial response; SD, stable disease; NA, not applicable.

${ }^{a}+$ indicates that patient is still alive and disease-free.

these three patients experienced any toxicity. The commencement of the second phase of treatment was delayed in seven patients $(70 \%)$, with a median delay of only five days (range 2-41 days). Dose delays were generally due to logistical factors such as transportation difficulties for rural patients, bed shortages, and delays to accommodate weekends and public holidays. There was no clear relationship between age and performance status or toxicity and dose density.

Chemotherapy was delayed in two patients because of grade 2 mucositis. While hospitalized, one of these patients suffered a worsening of his pre-existing confusional status. Both patients had further cycles of chemotherapy at the same dose without any difficulty when given an increase in the folinic acid rescue dose. A reduction in the glomerular filtration rate led to a prophylactic dose reduction in patients when the rate was $<55 \%$ of normal.

\section{Response Rates and Overall Survival}

Table 2 also summarizes the response to therapy, time to progression, and overall survival for this cohort. The median follow-up was 23 months (range 4-43 months). Six patients had a complete response and three patients achieved a partial response, giving an overall response rate of $90 \%$ (95\% confidence interval, $56 \%-100 \%$ ). Overall, rapid clinical responses were observed, often after one cycle of HDMTX. The median time to response was two months (1-4 months).

Eight of the ten patients continued onto the second phase of the treatment protocol, but only four patients went onto the third phase. Two patients did not proceed beyond the first phase because of lack of radiologic improvement for one and lack of clinical improvement for the other. Indeed, the second patient, with a history of steroid-responsive dementia over several years' duration, stopped treatment despite a complete response on radiologic grounds because there was no improvement in his mental state. Interestingly, his dysphasia and mobility improved significantly over the following months. Of the four patients who completed only the second phase of therapy, two ceased treatment because of radiologic documented progression subsequent to an initial response to therapy. One rural patient opted not to travel into the city for further therapy after achieving a complete response, and one patient elected to stop treatment because of grade 2 mucositis despite achieving a complete response.

Of the six patents who attained a complete response, four progressed at a median of 18.5 months after initial therapy $(15,18,19$, and 24 months). All four were retreated with HDMTX (range 1-6 cycles) and tolerated retreatment well. Two of the four attained a second complete response to HDMTX. One continues to be diseasefree after a further 15 months of follow-up, the other developed multifocal relapse seven months after retreatment. The remaining two patients did not demonstrate any response to retreatment. Two of the three patients who only attained a partial response to therapy went on to receive whole-brain radiotherapy at progression. One of these patients survived 20 months after radiotherapy, and the other remains disease-free at eight months' follow-up. The third patient is still undergoing salvage chemotherapy.

The median time to progression for the cohort was 18 months (range 2-24 months); the median overall survival for the cohort was 36 months (range 4-43 months). If a complete response was attained, the overall survival of this subgroup was a mean of 43 months. All patients maintained or improved their performance status.

\section{Discussion}

The incidence of PCNSL is rising and commonly affects men and women in their sixth and seventh decades (Rosenthal and Green, 1995). Standard treatment practice includes surgical biopsy for diagnosis, corticosteroids as adjunctive therapy in the initial phases of treatment, 
and definitive therapy with radiotherapy, chemotherapy, or a combination of both modalities. Chemotherapeutic strategies remain contentious, with options including multi-agent or single-agent regimens, intrathecal or i.v. administration, and blood-brain barrier disruption. The role of postchemotherapy whole-brain irradiation is also unclear (Rosenthal and Green, 1995).

Most published series do contain some patients older than 60 years; however, they usually represent a minority of the group. There are few reports with substantial numbers of patients older than 70 years; indeed, many analyses only include patients younger than 70 years. The older than 70 years group is often unrepresented or constitutes $<20 \%$ of the study population (Brada et al., 1990; Cher et al., 1996; DeAngelis et al., 1992; Neuwelt et al., 1991; Rosenthal and Green, 1995; Rosenthal et al., 1993). In contrast, our entire patient group was older than 60 years, and eight of ten $(80 \%)$ were older than 70 years. Furthermore, six of the ten patients $(60 \%)$ had an ECOG performance status of two or three.

HDMTX is an established single-agent regimen for PCNSL (Cher et al., 1996; Freilich et al., 1996). Blay et al. demonstrated that HDMTX was the only treatment factor that was of independent prognostic value in a multivariate analysis of 226 patients treated with various chemotherapy and radiotherapy regimens (Blay et al., 1998). The same study found that there was no evidence of an increased incidence of late neurotoxicity with HDMTX. Some authors have argued that wholebrain irradiation may be deferred indefinitely after HDMTX in an attempt to minimize treatment-related neurotoxicity that may be even more common in this elderly population (Abrey et al., 1998; Cher et al., 1996; Freilich et al., 1996).

Our study provides insights into the feasibility and efficacy of using HDMTX as first-line treatment for PCNSL in an elderly population. HDMTX is well tolerated in this group of patients and has a comparable response rate to that found in other series using HDMTX. The response rate in our patients was $90 \%$, with a complete response rate of $60 \%$. This compares with response rates reported in other series ranging from 50\%-100\% (Blay et al., 1998; Cher et al., 1996; Freilich et al. 1996; Gabbai et al., 1989). Progression-free and overall survival of 18 months and 36 months is comparable with many other chemotherapy studies, and some longer-term survivors have been seen (Rosenthal and Green, 1995). Importantly, all patients maintained or improved their performance status after therapy.

Interestingly, two of four patients retreated with HDMTX at relapse achieved a second complete response. This outcome from HDMTX therapy may be considered surprising because the age group older than 60 years has consistently had a poorer prognosis than younger patients (Blay et al., 1998; Nelson et al., 1992; O’Neill et al., 1999).

This study also demonstrates the tolerability of HDMTX in this elderly patient population. No grade IV toxicity or episodes of febrile neutropenia were seen. The toxicities observed were self-limiting and caused little inconvenience to the patients concerned. Importantly, we were able to achieve excellent dose intensity in almost all patients. Three patients required a dose reduction because of a decline in renal function. Treatment delays were predominantly related to logistical issues rather than toxicity.

In summary, this study demonstrated that HDMTX is well tolerated in an elderly population with PCNSL. Furthermore, this single-agent regimen results in high response rates and in a median survival benefit comparable with other chemotherapy or radiotherapy regimens. The incidence of PCNSL in the immunocompetent is rising, and many of these patients are elderly. Thus, consideration needs to be given to treatment protocols for this population. HDMTX offers an effective and safe therapy for elderly patients with PCNSL.

\section{References}

Abrey, L.E., DeAngelis, L.M., and Yahalom, J. (1998) Long-term survival in primary CNS lymphoma. J. Clin. Oncol. 16, 859-863.

Berry, M.P., and Simpson, W.J. (1981) Radiation therapy in the management of primary malignant lymphoma of the brain. Int. J. Radiat. Oncol. Biol. Phys. 7, 55-59.

Blay, J.Y., Conroy, T., Chevreau, C., Thyss, A., Quesnel, N., Eghbali, H., Bouabdallah, R., Coiffier, B., Wagner, J.P., Le Mevel, A., Dramais-Marcel, D., Baumelou, E., Chauvin, F., and Biron, P. (1998) High dose methotrexate for the treatment of primary cerebral lymphomas: Analysis of survival and late neurologic toxicity in a retrospective series. J. Clin. Oncol. 16, 864-871.

Brada, M., Dearnaley, D., Horwich, A., and Bloom, H.J.G. (1990) Management of primary cerebral lymphoma with initial chemotherapy: Preliminary results and comparison with patients treated with radiotherapy alone. Int. J. Rad. Oncol. Biol. Phys. 18, 787-792.

Cher, L., Glass, J., Griffith, G.R., and Hochberg, F.H. (1996) Therapy of primary CNS lymphoma with methotrexate-based chemotherapy and deferred radiotherapy: Preliminary results. Neurology 46, 1757-1759.

DeAngelis, L.M., Yahalom, J., Thaler, H.T., and Kher, U. (1992) Combined modality therapy for primary CNS lymphoma. J. Clin. Oncol. 10, $635-643$
Eby, N.L., Grufferman, S., Flannelly, C.M., Schold, S.C., Jr., Vogel, F.S., and Burger, P.C. (1988) Increasing incidence of primary brain lymphoma in the U.S. Cancer 62, 2461-2465.

Fine, H.A., and Mayer, R.J. (1993) Primary central nervous system lymphoma. Ann. Int. Med. 119, 1093-1104.

Freilich, R.J., Delattre, J.Y., Monjour, A., and DeAngelis, L.M. (1996) Chemotherapy without radiation therapy as initial treatment for primary CNS lymphoma in older patients. Neurology 46, 435-439.

Gabbai, A.A., Hochberg, F.H., Linggood, R.M., Bashir, R., and Hotleman, K. (1989) High dose methotrexate for non-AIDS primary central nervous system lymphoma. J. Neurosurg. 70, 190-194.

Lutz, J.M, and Coleman, M.P. (1994) Trends in primary cerebral lymphoma. Br. J. Cancer 70, 716-718.

Murray K., Kun L., and Cox J. (1986) Primary malignantlymphoma of the central nervous system: Results of treatment of 11 cases and review of the literature. J. Neurosurg. 65, 600-607.

Nelson, D.F., Martz, K.L., Bonner, H., Nelson, J.S., Newall, J., Kerman, H.D., Thomson, J.W., and Murray, K.J. (1992) Non-Hodgkin's lymphoma of the brain: Can high dose, large volume radiation therapy improve survival? Report on a prospective trial by the Radiation Therapy 
S. Ng et al.: HDMTX in elderly patients with PCNSL

Oncology Group (RTOG): RTOG 8315. Int. J. Radiat. Oncol. Biol. Phys. 23, 9-17.

Neuwelt, E.A., Goldman, D.L., Dahlborg, S.A., Crossen, J., Ramsey, F., RomanGoldstein, S., Braziel, R., and Dana, B. (1991) Primary CNS lymphoma treated with osmotic blood-brain barrier disruption: Prolonged survival and preservation of cognitive function. J. Clin. Oncol. 9, 1580-1590.

Oken, M.M., Creech, R.H., Tormey, D.C., Horton, J., Davis, T.E., McFadden, E.T., and Carbone, P.P. (1982) Toxicity and response criteria of the Eastern Cooperative Oncology Group. Am. J. Clin. Oncol. 5, 649-655.

O'Neill, B.P., Wang, C.H., O'Fallon, J.R., Colgan, J.D., Earle, J.D., Krigel, R.L., Brown, L.D., and McGinnis, W.L. (1999) Primary central nervous system non-Hodgkin's lymphoma (PCNSL): Survival advantages with combined therapy? A final report of the North Central Cancer Treatment Group (NCCTG) Study 86-72-52. Int. J. Radiat. Oncol. Biol. Phys. 43, 559-563.

Rosenthal, M.A., Sheridan W.P., Green, M.D., Liew, K., and Fox, R.M. (1993) Primary cerebral lymphoma: An argument for the use of adjunctive systemic chemotherapy in treatment. Aust. N. Z. J. Surg. 63, 30-32.

Rosenthal, M.A., and Green, M.D. (1995) Cerebral lymphoma. In: Kaye, A.H., and Laws, E.R., Jr. (Eds.), Brain Tumors. Edinburgh: Churchill Livingstone. pp. 841-869.

Singh, A., Strobos, R.J., Singh, B.M., Rothballer, A.B., Reddy, V., Puljic, S., and Poon, T.P. (1982) Steroid-induced remissions in CNS lymphoma. Neurology 32, 1267-1271. 